\title{
SOME INEQUALITIES FOR MAXIMUM MODULES OF POLYNOMIALS
}

\author{
N.K. GOVIL \\ Department of Algebra, Combinatorics \& Analysis \\ Division of Mathematics \\ Auburn University, Auburn, AL \\ (Received March 29, 1990 and in revised form August 20, 1990)
}

\begin{abstract}
A well-known result of Ankeney and Rivlin states that if $p(z)$ is a polynomial of degree $n$, such that $p(z) \neq 0$ in $|z|<1$, then $\max _{|z|=R \geq 1}|p(z)| \leq\left(\frac{R^{n}+1}{2}\right) \max _{|z|=1}|p(z)|$. In this paper we prove 'some generalizations and refinements of this result.
\end{abstract}

KEYWORDS AND PHRASES. Inequalities in the complex domain, polynomials, extremal problems. 1980 AMS SUBJECT CLASSIFICATION CODE. 30A10.

\section{INTRODUCTION AND STATEMENT OF RESULTS.}

Let $p(z)=\sum_{\nu=0}^{n} a_{\nu} z^{\nu}$ be a polynomial of degree $n$, and let $M(p, r)=\max _{|z|=r}|p(z)|$. The following result concerning the size of $M(p, r)$ is well known.

THEOREM A. (S. Bernstein, cf. [1]) If $p(z)=\sum_{\nu=0}^{n} a_{\nu} z^{\nu}$ is a polynomial of degree $n$, then

$$
M(p, R) \leq R^{n} M(p, 1) \text { for } R \geq 1
$$

with equality only for $p(z)=\lambda z^{n}$.

For polynomials not vanishing in $|z|<1$, Ankeney and Rivlin [2] proved

THEOREM B. [2] If $p(z)=\sum_{\nu=0}^{n} a_{\nu} z^{\nu}$ is a polynomial of degree $n, p(z) \neq 0$ in $|z|<1$, then

$$
M(p, R) \leq\left(\frac{R^{n}+1}{2}\right) M(p, 1) \text { for } R \geq 1
$$

The result is best possible with equality only for the polynomial $p(z)=\left(\lambda+\mu z^{n}\right),|\lambda|=|\mu|$.

Although the above result is best possible but still it seems very natural to ask the following questions in connection with the above theorem. 
Q.1 What is the bound in (1.2) if we replace the condition $p(z) \neq 0$ in $|z|<1$ by $p(z) \neq 0$ in $|z|<K, K \geq 1$ or more generally if $p(z)=a_{n} \prod_{\nu=1}^{n}\left(z-z_{\nu}\right)$, where $\left|z_{\nu}\right| \geq K_{\nu} \geq 1$ ?

Q.2 Since (1.2) becomes equality only for polynomials $p(z)=\lambda+\mu z^{n},|\lambda|=|\mu|$, is it possible to obtain a sharper bound in (1.2) if not all the coefficients $a_{1}, a_{2}, \ldots, a_{n-1}$ in the polynomial $p(z)=\sum_{\nu=0}^{n} a_{\nu} z^{\nu}$ are zero? More generally, is it possible to obtain a bound in (1.2) which depends as well on some or all the coefficients $a_{1}, a_{2}, \ldots, a_{n-1}, a_{n}$ ?

In an attempt to answer these questions, we prove

THEOREM Let $p(z)=a_{n} \prod_{\nu=1}^{n}\left(z-z_{\nu}\right), a_{n} \neq 0$, be a polynomial of degree $n \geq 2$, and let $\left|z_{\nu}\right| \geq K_{\nu} \geq$ $1,1 \leq \nu \leq n$. Then

$$
\begin{aligned}
& M(p, R) \leq \frac{\left(R^{n}+1\right)}{2}[\left.1-\left(\frac{R^{n}-1}{R^{n}+1}\right) \frac{1}{1+\frac{2}{n} \sum_{\nu=1}^{n} \frac{1}{K_{\nu}-1}}\right] M(p, 1) \\
&-\left|p^{\prime}(0)\right|\left(\frac{R^{n}-1}{n}-\frac{R^{n-2}-1}{n-2}\right), \text { if } n>2
\end{aligned}
$$

and

$$
M(p, R) \leq \frac{\left(R^{2}+1\right)}{2}\left[1-\left(\frac{R^{2}-1}{R^{2}+1}\right) \frac{\left(K_{1}-1\right)\left(K_{2}-1\right)}{\left(K_{1} K_{2}-1\right)}\right] M(p, 1)-\left|p^{\prime}(0)\right| \frac{(R-1)^{2}}{2}
$$

if $n=2$.

Since for $R>1, \frac{\left(R^{x}-1\right)}{x}$ is an increasing function of $x$, the expression $\left|p^{\prime}(0)\right|\left(\frac{R^{n}-1}{n}-\frac{R^{n-2}-1}{n-2}\right)$ is always nonnegative. Thus for polynomials of degree greater than 1, our Theorem generalizes and sharpens Theorem B due to Ankeney and Rivlin [2]. (The case when the polynomial $p(z)$ is of degree 1 is uninteresting because then $M(P, R)$ can be calculated trivially). In fact excepting the case when the polynomial $p(z)$ has zeros on $|z|=1$ and $p^{\prime}(0)=0$, the bound obtained by our Theorem is always sharper than the bound obtained by Theorem B.

Remark: The statement of our Theorems might suggest that we need to know all the zeros of the polynomial in order to apply our theorem but this is not so. No doubt, the usefulness of the theorem will be heightened if the polynomial is given in terms of its zeros. If in particular, we know that the polynomial $p(z)$ is product of two or more polynomials having zeros in $|z| \geq K_{1}>1,|z| \geq K_{2}>1$, etc. each of norm $\leq 1$ (here $\|p\|=M(p, 1)$ ) then $p(z)$ would be of norm $\leq 1$, and we would have a better estimte for $M(p, R)$ by using our Theorem than one would get from Theorem B.

If the polynomial $p(z)$ has no zeros in $|z|<K, K \geq 1$, we get from our Theorem, the following

COROLLARY 1 If $p(z)=\sum_{\nu=0}^{n} a_{\nu} z^{\nu}$ is a polynomial of degree $n \geq 2$, having no zeros in $|z|<$ $K, K \geq 1$, then

$$
M(p, R) \leq\left(\frac{R^{n}+K}{1+K}\right) M(p, 1)-\left|a_{1}\right|\left(\frac{R^{n}-1}{n}-\frac{R^{n-2}-1}{n-2}\right) \text { if } n>2
$$

and 


$$
M(p, R) \leq\left(\frac{R^{2}+K}{1+K}\right) M(p, 1)-\left|a_{1}\right| \frac{(R-1)^{2}}{2} \text { if } n=2 .
$$

In particular, if we take $K=1$, we get

COROLLARY 2 If $p(z)=\sum_{\nu=0}^{n} a_{\nu} z^{\nu}$ is a polynomial of degree $n \geq 2$, having no zeros in $|z|<1$, then

$$
M(p, R) \leq\left(\frac{R^{n}+1}{2}\right) M(p, 1)-\left|a_{1}\right|\left(\frac{R^{n}-1}{n}-\frac{R^{n-2}-1}{n-2}\right), \text { if } n>2
$$

and

$$
M(p, R) \leq\left(\frac{R^{2}+1}{2}\right) M(p, 1)-\left|a_{1}\right| \frac{(R-1)^{2}}{2} \text { if } n=2 .
$$

Equality in (1.7) and (1.8) holds for $p(z)=\left(\lambda+\mu z^{n}\right),|\lambda|=|\mu|$.

Inequality (1.7) also appears in [4, Lemma 4].

For polynomials of degree greater 1, clearly Corollaries 1 and 2 provide a generalization and refinement of Theorem B.

If in (1.5) and (1.6) we divide both the sides by $R^{n}$ and make $R \rightarrow \infty$, we get

COROLLARY 3 If $p(z)=\sum_{\nu=0}^{n} a_{\nu} z^{\nu}$ is a polynomial of degree $n \geq 2$, having no zeros in $|z|<$ $K, K \geq 1$, then

$$
\left|a_{n}\right|+\frac{\left|a_{1}\right|}{n} \leq\left(\frac{1}{1+\hbar}\right) M(p, 1)
$$

Taking $K=1$ in the above corollary, we get

COROLLARY 4 If $p(z)=\sum_{\nu=0}^{n} a_{\nu} z^{\nu}$ is a polynomial of degree $n$ having no zeros in $|z|<1$, then

$$
\left|a_{n}\right|+\frac{\left|a_{1}\right|}{n} \leq \frac{1}{2} M(p, 1)
$$

The result is best possible with equality for the polynomial $p(z)=\left(\lambda+\mu z^{n}\right),|\lambda|=|\mu|$.

Inequality (1.10) is obviously an improvement over the known result

$$
\left|a_{n}\right| \leq \frac{1}{2} M(p, 1)
$$

and can also be obtained by applying Vissar's inequality [4] to the polynomial $p^{\prime}(z)$. 


\section{LEMMAS}

LEMMA 1 If $p(z)=a_{n} \prod_{\nu=1}^{n}\left(z-z_{\nu}\right), a_{n} \neq 0$ is a polynomial of degree $n,\left|z_{\nu}\right| \geq K_{\nu} \geq 1$ for $1 \leq \nu \leq n$, then

$$
M\left(p^{\prime}, 1\right) \leq n\left\{\left(\sum_{\nu=1}^{n} \frac{1}{K_{\nu}-1}\right) /\left(\sum_{\nu=1}^{n} \frac{K_{\nu}+1}{K_{\nu}-1}\right)\right\} M(p, 1) .
$$

Here and elsewhere $M\left(p^{\prime}, 1\right)$ stands for $\max _{|z|=1}\left|p^{\prime}(z)\right|$. In $(2.1)$, equality holds for the polynomial $p(z)=$ $(z+K)^{n}, K \geq 1$

Above result is due to Govil and Labelle [5].

LEMMA 2 Let $p(z)=\sum_{\nu=0}^{n} a_{\nu} z^{\nu}$ be a polynomial of degree $n, n \geq 2$. Then for all $R>1$,

$$
M(p, R) \leq R^{n} M(p, 1)-\left(R^{n}-R^{n-2}\right)|p(0)| .
$$

The coefficient of $|p(0)|$ is the best possible for each $R$.

Above result is due to Frappier et al [6, Theorem 2].

LEMMA 3 If $p(z)=a_{n} \prod_{\nu=1}^{n}\left(z-z_{\nu}\right), a_{n} \neq 0$ is a polynomial of degree $n>2,\left|z_{\nu}\right| \geq K_{\nu} \geq 1$ for $1 \leq \nu \leq n$ then for $R>1,0 \leq \phi \leq 2 \pi$, we have

$$
\begin{array}{r}
\left|p^{\prime}\left(R e^{\imath \phi}\right)\right| \leq n R^{n-1}\left\{\left(\sum_{\nu=1}^{n} \frac{1}{K_{\nu}-1}\right) /\left(\sum_{\nu=1}^{n} \frac{K_{\nu}+1}{K_{\nu}-1}\right)\right\} M(p, 1) \\
-\left(R^{n-1}-R^{n-3}\right)\left|p^{\prime}(0)\right|
\end{array}
$$

Proof of Lemma 3. Since $p(z)$ is a polynomial of degree $>2$, the polynomial $p^{\prime}(z)$ is of degree $\geq 2$, hence on applying Lemma 2 to the polynomial $p^{\prime}(z)$, we get

$$
\left|p^{\prime}\left(R e^{i \phi}\right)\right| \leq R^{n-1} M\left(p^{\prime}, 1\right)-\left(R^{n-1}-R^{n-3}\right)\left|p^{\prime}(0)\right|,
$$

which when combined with Lemma 1 , gives Lemma 3.

\section{PROOF OF THE THEOREM}

For each $\phi, \quad 0 \leq \phi<2 \pi$, we have

$$
p\left(R e^{\imath \phi}\right)-p\left(e^{\imath \phi}\right)=\int_{1}^{R} e^{i \phi} p^{\prime}\left(r e^{\imath \phi}\right) d r
$$

Hence

$$
\left|p\left(R e^{\imath \phi}\right)-p\left(e^{\imath \phi}\right)\right| \leq \int_{1}^{R}\left|p^{\prime}\left(r e^{\imath \phi}\right)\right| d r
$$

which when combined with Lemma 3 gives 


$$
\begin{aligned}
& \left|p\left(R e^{\imath \phi}\right)-p\left(e^{\imath \phi}\right)\right| \leq n\left\{\left(\sum_{\nu=1}^{n} \frac{1}{K_{\nu}-1}\right) /\left(\sum_{\nu=1}^{n} \frac{K_{\nu}+1}{K_{\nu}-1}\right)\right\} M(p, 1) \int_{1}^{R} r^{n-1} d r \\
& -\int_{1}^{R}\left(r^{n-1}-r^{n-3}\right) d r\left|p^{\prime}(0)\right| \\
& =\left(R^{n}-1\right)\left\{\left(\sum_{\nu=1}^{n} \frac{1}{K_{\nu}-1}\right) /\left(\sum_{\nu=1}^{n} \frac{K_{\nu}+1}{K_{\nu}-1}\right)\right\} M(p, 1) \\
& -\left(\frac{R^{n}-1}{n}-\frac{R^{n-2}-1}{n-2}\right)\left|p^{\prime}(0)\right| \\
& =\frac{\left(R^{n}-1\right)}{\left(1+\frac{\sum_{\nu=1}^{n} \frac{K_{\nu}}{K_{\nu}-1}}{\sum_{\nu=1}^{n} \frac{1}{K_{\nu}-1}}\right.} M I(p, 1)-\left(\frac{R^{n}-1}{n}-\frac{R^{n-2}-1}{n-2}\right)\left|p^{\prime}(0)\right| \\
& =\frac{\left(R^{n}-1\right)}{\left(\frac{n+\sum_{\nu=1}^{n} \frac{1}{K_{\nu}-1}}{\sum_{\nu=1}^{n} \frac{T_{\nu}-1}{K_{\nu}}}+1\right)} M(p, 1)-\left(\frac{R^{n}-1}{n}-\frac{R^{n-2}-1}{n-2}\right)\left|p^{\prime}(0)\right| \\
& =\frac{R^{n}-1}{\left\{2+n / \sum_{\nu=1}^{n} \frac{1}{\left(K_{\nu}-1\right)}\right\}} M(p, 1)-\left(\frac{R^{n}-1}{n}-\frac{R^{n-2}-1}{n-2}\right)\left|p^{\prime}(0)\right| \\
& =\frac{\left(R^{n}-1\right)}{2}\left\{1 /\left(1+\frac{1}{\frac{2}{n} \sum_{\nu=1}^{n} \frac{1}{K_{\nu}-1}}\right)\right\} M(p, 1)-\left(\frac{R^{n}-1}{n}-\frac{R^{n-2}-1}{n-2}\right)\left|p^{\prime}(0)\right| \\
& =\frac{\left(R^{n}-1\right)}{2}\left\{1-\frac{1}{1+\frac{2}{n} \sum_{\nu=1}^{n} \frac{1}{K_{\nu}-1}}\right\} M(p, 1)-\left(\frac{R^{n}-1}{n}-\frac{R^{n-2}-1}{n-2}\right)\left|p^{\prime}(0)\right|,
\end{aligned}
$$

which gives

$$
\begin{array}{r}
\left|p\left(R e^{i \phi}\right)\right| \leq \frac{\left(R^{n}-1\right.}{2}\left\{1-\frac{1}{1+\frac{2}{n} \sum_{\nu=1}^{n} \frac{1}{K_{\nu}-1}}\right\} M(p, 1)+M(p, 1) \\
-\left(\frac{R^{n}-1}{n}-\frac{R^{n-2}-1}{n-2}\right)\left|p^{\prime}(0)\right| \\
=\left\{\frac{R^{n}+1}{2}-\frac{\left(R^{n}-1\right) / 2}{1+\frac{2}{n} \sum_{\nu=1}^{n} \frac{1}{K_{\nu}-1}}\right\} M(p, 1)-\left(\frac{R^{n}-1}{n}-\frac{R^{n-2}-1}{n-2}\right)\left|p^{\prime}(0)\right| \\
=\frac{\left(R^{n}+1\right)}{2}\left\{1-\left(\frac{R^{n}-1}{R^{n}+1}\right)\left(\frac{1}{1+\frac{2}{n} \sum_{\nu=1}^{n} \frac{1}{K_{\nu}-1}}\right)\right\} M(p, 1) \\
-\left|p^{\prime}(0)\right|\left(\frac{R^{n}-1}{n}-\frac{R^{n-2}-1}{n-2}\right),
\end{array}
$$

which is (1.3).

The proof of (1.4) follows on the same lines as the proof of (1.3) but instead of Lemma 3 the corresponding result for polynomials of degree 2 , which is

$$
\left|p^{\prime}\left(R e^{\imath \phi}\right)\right| \leq R\left\{1-\frac{\left(K_{1}-1\right)\left(K_{2}^{\prime}-1\right)}{\left(K_{1} K_{2}-1\right)}\right\} M(p, 1)-(R-1)\left|p^{\prime}(0)\right| .
$$

We omit the details.

\section{REFERENCES}

1. POLYA, G. and SZEGö, S. G., Aufgaben und Lehrsatze aus der Analysis, Vol, 1, p. 137, Problem 269, New York 1945.

2. ANKENEY, N. C. and RIVlin, T. J., On a theorem of S. Bernstein, Pacific J. Math. 5 (1955), 849-852. 
3. GOVIL, N. K. Inequalities for the derivative of a polynomial, J. Approx. Theory, to appear.

4. VISSER, C., A simple proof of certain inequalities concerning polynomials, Nederl. Akad. Wetensch. Proc. 47, 276-281 = Indag. Math. 7 (1945), 81-86.

5. GOVIL, N. K. and LABELle, G., On Bernstein's Inequality, J. Math. Anal. Appl. 126 (1987), 494-500.

6. FRAPPIER, C., RAHMAN, Q. I. and RUSCHEWEYH, S., New Inequalities for polynomials, Trans, Amer. Math. Soc. 28 (1) (1958), 69-99. 


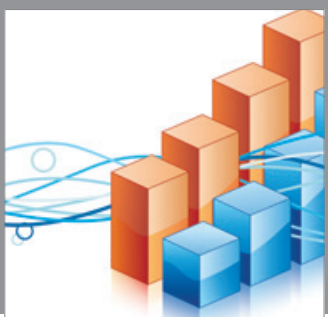

Advances in

Operations Research

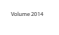

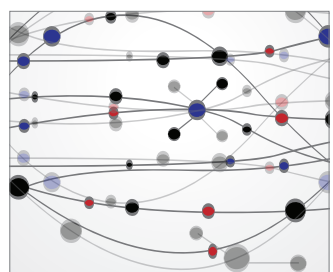

\section{The Scientific} World Journal
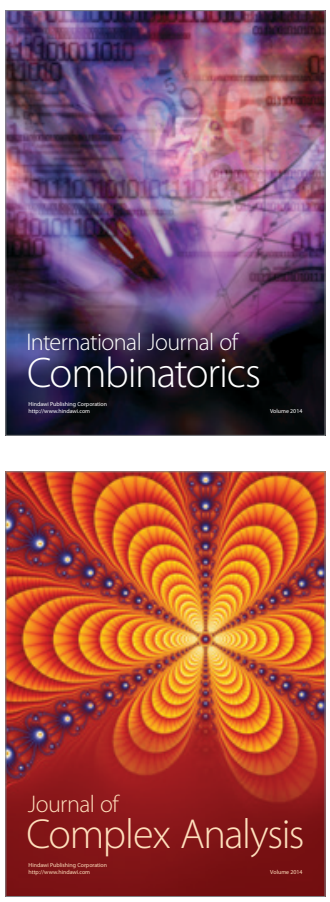

International Journal of

Mathematics and

Mathematical

Sciences
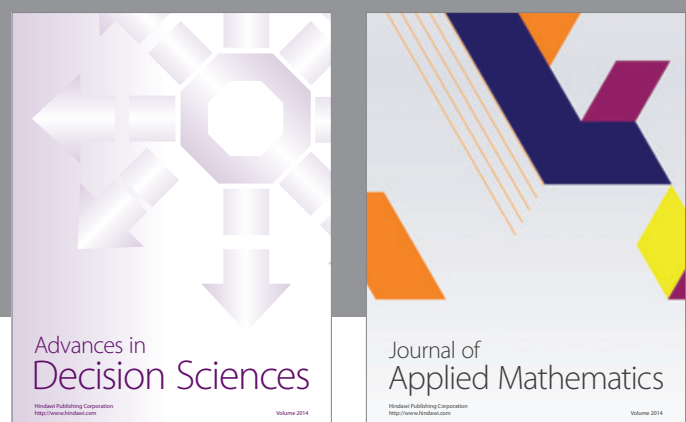

Journal of

Applied Mathematics
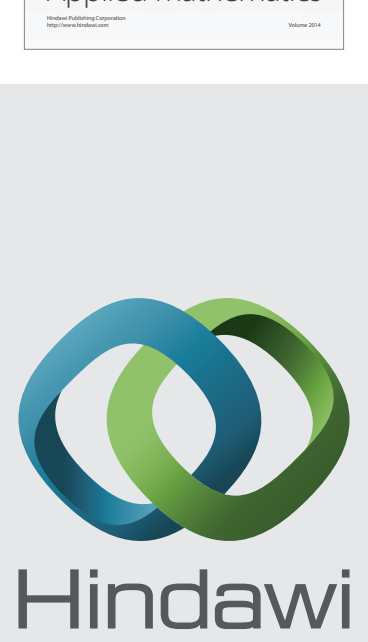

Submit your manuscripts at http://www.hindawi.com
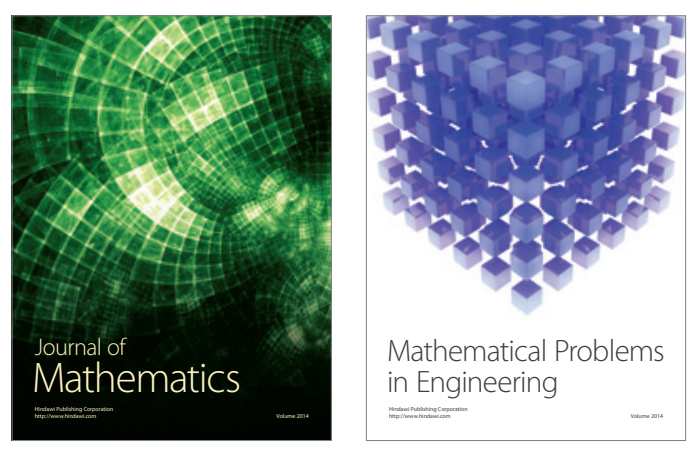

Mathematical Problems in Engineering
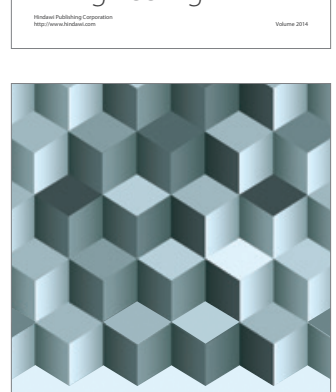

Journal of

Function Spaces
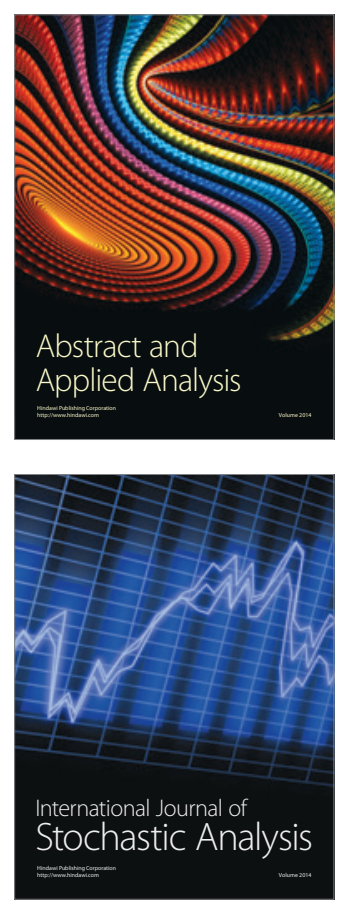

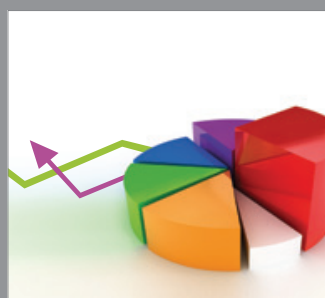

ournal of

Probability and Statistics

Promensencen
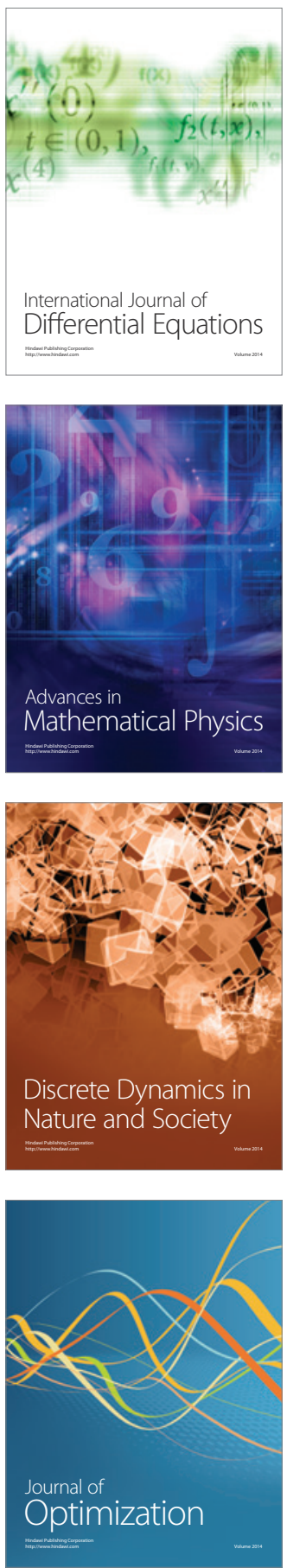